Sina Bartfeld, Hannah Schickl, Anja Pichl, Angela Osterheider und Lilian Marx-Stölting

\title{
2. Organoide in Forschung und Anwendung: eine Einführung
}

\subsection{Einführung in die Organoidforschung und den Themenband}

\subsubsection{Was sind Organoide?}

Organoide sind aus Stammzellen oder Vorläuferzellen in vitro erzeugte, dreidimensionale Strukturen aus Zellen. Sie ähneln Organen in vivo in Hinblick auf die enthaltenen Zelltypen, deren räumliche Anordnung und spezifische Funktionsfähigkeit. Ihre Entstehung wird häufig mit dem Begriff „Selbstorganisation“ charakterisiert. Darunter versteht man einen (bislang nur unzureichend verstandenen) Prozess der Bildung komplexer Strukturen aus Ausgangszellen durch Interaktionen der Zellen untereinander sowie zwischen den Zellen und ihrer Umgebung (siehe Lewis/Keshara/Kim/Grapin-Botton et al., Kap. 3.2). Stammzellen sind Zellen, die durch Teilung sowohl weitere Stammzellen (Fähigkeit zur Selbsterneuerung) als auch spezialisierte Zellen (Fähigkeit zur Differenzierung) hervorbringen können. ${ }^{1}$ Vorläuferzellen sind hingegen bereits auf die Bildung bestimmter Zelltypen festgelegte Nachkommen von Stammzellen.

Die Bandbreite der Organe, die auf diese Weise in unterschiedlichen Spezies (und zum Teil auch in Kombination verschiedener Spezies) nachgebildet werden können, ist inzwischen sehr groß und umfasst Organe und Zelltypen, die aus den Zellen aller drei Keimblätter entstanden sind. Keimblätter nennt man die drei Zellschichten (Entoderm/Innenschicht, Mesoderm/Mittelschicht, Exoderm/Außenschicht), die sich während der Embryonalentwicklung bilden und die im Verlauf der Weiterentwicklung unterschiedliche Gewebe hervorbringen können. Die drei Keimblätter enthalten sozusagen die entwicklungsgeschichtlich am weitesten voneinander entfernten Gruppen von Zellen. Da die Organoidtechnologie bereits diese größtmöglichen zellulären Un-

1 Eine aktuelle Übersicht zur Stammzellforschung bietet der Themenband der IAG Gentechnologiebericht (Zenke et al., 2018). Darin enthalten ist auch eine kurze Beschreibung der Organoidtechnologie (Bartfeld/Clevers, 2018). 
terschiede nachbilden kann, wird angenommen, dass grundsätzlich Organoide aller Organe hergestellt werden können. Zur Vielzahl der Organe, hinsichtlich derer bereits Organoide erzeugt wurden, zählen u. a.: Darm (siehe Interview mit Clevers, Kap. 2.2; Kayisoglu/Schlegel/Bartfeld, Kap. 3.8); Gehirn (siehe Tanaka/Park, Kap. 3.5); Magen, Pankreas, Leber, Prostata, Speiseröhre, Gallenblase, Schilddrüse, Lunge (siehe Frum/ Spence, Kap. 3.1 und Lewis/Keshara/Kim/Grapin-Botton, Kap. 3.2); Nieren (siehe Gupta/Dilmen/Morizane, Kap. 3.6), der weibliche Reproduktionstrakt (siehe Chumduri/Turco, Kap. 3.7) und vielen weiteren, Tendenz steigend. Dabei steht jedoch nicht immer ein Organoid für das gesamte Organ: Häufig bilden mehrere Organoide unterschiedliche Aspekte einzelner Organe nach und machen sie dadurch der experimentalwissenschaftlichen Forschung zugänglich (siehe auch wissenschaftstheoretische Überlegungen von Fagan, Kap. 4).

Ihr Anwendungspotenzial für verschiedene Bereiche, insbesondere der biomedizinischen Forschung und Therapie, ist vielversprechend. Dieses reicht von der Grundlagenforschung, beispielsweise zur In-vitro-Untersuchung der Organentstehung und zur Erforschung von Krankheiten, über die Nutzung als Testsysteme für die Medikamentenentwicklung und Toxizitätsprüfungen, bis hin zu Zell-, Gewebe- und Organersatz innerhalb der regenerativen Medizin. Gerade für das Bestreben einer zunehmend personalisierten Medizin weckt die Forschung an Organoiden große Hoffnungen und eröffnet neue Perspektiven.

\subsubsection{Gewinnung von Organoiden}

Organoide können entweder aus gewebespezifischen (adulten) Stammzellen oder aus pluripotenten Stammzellen hergestellt werden. Zu Letzteren zählen embryonale Stammzellen (ES-Zellen), die aus Embryonen gewonnen wurden, oder induzierte pluripotente Stammzellen (iPS-Zellen), die durch die „Reprogrammierung“ von Körperzellen (somatischen Zellen) erzeugt werden. Man kann daher auch von zwei unterschiedlichen „Organoidtechnologien“ sprechen und damit wesentliche Unterschiede zwischen auf adulten und pluripotenten Stammzellen basierenden Organoiden bzw. ihrer Herstellung verdeutlichen (vgl. z. B. Interview mit Clevers, Kap. 2.2). Grundzüge der Herstellung von Organoiden aus pluripotenten und adulten Stammzellen lassen sich folgendermaßen skizzieren:

Dass humane ES- und iPS-Zellen (hES- bzw. hiPS-Zellen) pluripotent sind, bedeutet, dass sie alle der über 200 möglichen Zelltypen des menschlichen Körpers bilden können - eine Fähigkeit, die hoch komplex und von einem aufeinander abgestimmten Zusammenspiel verschiedener Signale der zellulären Umgebung und deren Umsetzung 
innerhalb der Zelle abhängig ist. Solche Signale steuern u. a. die Morphogenese (Entwicklung), Gewebestruktur und Induktion von Organvorläufern während der Embryonalentwicklung und sind entscheidend für die kontinuierliche Entwicklung von Geweben aller drei Keimblätter. Für die Herstellung von Organoiden wird das Wissen über diese entwicklungsbiologischen Mechanismen genutzt und durch neue Experimente auch weiter vermehrt (siehe Frum/Spence, Kap. 3.1). Dabei werden durch Zugabe bestimmter Faktoren zur Nährlösung der pluripotenten Stammzellen die Signale, die die Zellen natürlicherweise im Körper bekommen, imitiert und dadurch verschiedene Entwicklungsschritte in der Petrischale initiiert. Durch die jeweilige Zusammensetzung der Nährlösung wird eine Vermehrung der Stammzellen oder eine gerichtete Differenzierung angeregt, d. h. eine zunehmende Spezialisierung auf die Bildung bestimmter Zelltypen. Dabei durchlaufen die Stammzellen den Differenzierungsprozess schrittweise. Die Zellen werden bei jedem Schritt näher an den gewünschten Zelltyp herangeführt und das Vermögen der Bildung anderer Zelltypen immer weiter eingeschränkt (siehe Frum/Spence, 3.1). Werden die Zellen dabei in eine dreidimensionale Matrix eingebettet, die der natürlichen extrazellulären Matrix ähnelt, ermöglicht dies den Zellen, sich räumlich zu organisieren, es entstehen Organoide. Organoide aus pluripotenten Stammzellen weisen eine größere Vielfalt an Zelltypen auf als Organoide aus adulten Stammzellen, jedoch sind Erstere in der Regel weniger ausgereift und ähneln häufig fetalem Gewebe.

Adulte Stammzellen werden aus Spendermaterial, z. B. Gewebeproben von Patientinnen und Patienten, ${ }^{2}$ gewonnen und sind lediglich multipotent und somit auf die Bildung bestimmter Zelltypen festgelegt. Zur Entwicklung von Organoiden aus diesen Zellen wird die natürliche Zellumgebung in vivo nachgeahmt. Dies ist im Prinzip ähnlich wie bei Organoiden aus pluripotenten Stammzellen, aber da adulte Stammzellen bereits gewebespezifisch sind, müssen sie nicht erst eine gerichtete Differenzierung in bestimmte Zelltypen durchlaufen, sondern es muss nur die Umgebung im adulten Gewebe in vitro imitiert werden. Hierzu werden die adulten Stammzellen in einer dreidimensionalen Matrix und einer Nährlösung kultiviert, die ihre Aktivität unterstützt und erhält. Diese Methode ist deutlich schneller und die entstehenden Organoide enthalten einheitlichere Zelltypen als die aus pluripotenten Stammzellen abgeleiteten Organoide. Ein Beispiel: Organoide der Epithelien (Deckgewebe, die die Oberflächen

2 Im vorliegenden Themenband wurde bewusst nicht einheitlich gegendert, da die Wahl den jeweiligen Autorinnen und Autoren überlassen wurde, ob und in welcher Form sie gendern möchten. Die Herausgeberinnen und Herausgeber haben dabei auf Konsistenz innerhalb jedes Kapitels sowie auf Orthografie und Grammatik geachtet. 
des Körpers auskleiden) können aus adulten oder pluripotenten Stammzellen abgeleitet werden. Die aus adulten Stammzellen abgeleiteten Organoide enthalten reines Epithel, während die aus pluripotenten Stammzellen abgeleiteten Organoide Epithel, Mesenchym (Bindegewebszellen) und sogar auch Endothel (Zellen, die das Innere der Blutgefäße auskleiden) parallel enthalten. Dadurch sind die aus adulten Stammzellen abgeleiteten Organoide reduzierter und erlauben besonders differenzierte Analysen von bestimmten Zelltypen, wie eben des Epithels.

Humanen iPS-Zellen und adulten Stammzellen ist gemeinsam, dass sie besonders gut für Anwendungen und Forschung im Bereich der personalisierten Medizin geeignet sind, da sie patientenspezifisch aus Zellen zu behandelnder Patientinnen und Patienten generiert werden können.

\subsubsection{Anwendungsfelder von Organoiden}

Organoide ermöglichen die wissenschaftliche Erforschung menschlicher Entwicklung, Physiologie und Pathologie in einem zuvor nicht gekannten Ausmaß und Präzisionsniveau. Bisher haben Wissenschaftlerinnen und Wissenschaftler dies an Tiermodellen und zweidimensionalen menschlichen Zellkulturmodellen erforscht. Entsprechende Ansätze haben zu unzähligen wichtigen Entdeckungen geführt, weisen jedoch spezifische Grenzen auf: In-vivo-Tiermodelle sind ethisch nicht unbedenklich, kostenintensiv und zeitaufwendig, zudem bilden sie die menschliche Physiologie nur unvollkommen nach und ihre Komplexität kann die Bestimmung von Ursache und Wirkung in Experimenten erschweren. Herkömmliche humane 2-D-Zellkulturmodelle sind hingegen oft zu einfach, da sie häufig nur Zellen eines Zelltyps enthalten. Darüber hinaus werden 2-D-Zellkulturmodelle typischerweise aus Krebsgewebe von Patientinnen und Patienten gewonnen oder durch virale Onkogene in einen krebsähnlichen Zustand gebracht, was die unbegrenzte Vermehrung dieser Modelle in vitro ermöglicht, aber auch zu genomischer Instabilität und Unterschieden dieser Modelle im Vergleich zu ihren In-vivo-Gegenstücken führen kann.

Organoide können hingegen auch aus gesunden menschlichen Zellen generiert werden, enthalten viele der in einem Organ vorkommenden Zelltypen und weisen eine stabile Genotyp-Phänotyp-Beziehung sowie Aspekte der Architektur, Physiologie und Funktion des menschlichen Organs auf. Aus diesen Gründen können viele komplexe Vorgänge gut mit Organoiden erforscht werden. Zur durch Organoide ermöglichten Grundlagenforschung gehören die Untersuchung der Embryonalentwicklung, der Organentwicklung (Organogenese) und der Aufrechterhaltung der Organfunktion. Außerdem können Organoide als Krankheitsmodelle für die Erforschung sowohl gene- 
tischer Krankheiten als auch von Infektionskrankheiten genutzt werden. Es laufen bereits einige klinische Studien unter Verwendung von Organoiden. ${ }^{3}$ Da Organoide sowohl aus gesundem als auch aus erkranktem Gewebe hergestellt werden können, bieten sie eine Vielzahl an Anwendungsmöglichkeiten in der Grundlagen- und translationalen Forschung. Aufgrund des mangelnden Zugangs zu gesundem und erkranktem Gewebe von Patientinnen und Patienten bleibt jedoch auch das Interesse an Organoiden aus humanen pluripotenten Stammzellen erhalten, die erneuerbar und breit verfügbar sind.

Aktuell werden Organoide, insbesondere Lungen-, Nieren-, Leber-, Pankreas- und Darmorganoide, zur Erforschung von COVID-19 genutzt, insbesondere für die Modellierung bestimmter Krankheitsabläufe und für das Screening bestehender Medikamente für andere Krankheiten auf Wirksamkeit gegen Sars-Cov-2 (Clevers, 2020; Mallapaty, 2020). Auch für die Krebsforschung sind sie von Bedeutung (siehe Kretzschmar, Kap. 3.4). Wenn Krebsgewebe als Ausgangsmaterial für Organoide dient, lassen sich beispielsweise die spezifischen Eigenschaften des jeweiligen Tumors untersuchen.

Innerhalb der personalisierten Medizin können patienteneigene bzw. autologe Organoide dazu genutzt werden, verschiedene Medikamente vorab zu testen und das für die Patientin bzw. den Patienten am besten geeignete auszuwählen. Im Fall der Mukoviszidose werden aus Patientenzellen entwickelte Organoide bereits zu diesem Zweck genutzt. Diese prognostische Anwendung von Organoiden ist bereits Teil des niederländischen Gesundheitssystems (siehe Interview mit Clevers, Kap. 2.2). Es besteht die Hoffnung, dass in Zukunft dieses Prinzip auch für Krebspatientinnen und -patienten angewendet werden könnte. Erste Studien weisen darauf hin, dass die patienteneigenen Organoide in vitro bei Zugabe bestimmter Medikamente dieselbe Reaktion zeigen wie der Tumor in der Patientin bzw. im Patienten (siehe Kretzschmar, Kap. 3.4).

Auf dem Feld der regenerativen Medizin besteht die Hoffnung, dass humane Organoide in Zukunft auch transplantierbares Gewebe liefern und so dem Mangel an transplantierbaren Organen begegnen könnten. Aus eigenen Stammzellen hergestellte, also autologe, Organoide in Patientinnen und Patienten transplantieren zu können, wäre ein weiterer und großer Schritt hin zur personalisierten Medizin. Dabei könnten zudem eventuell vorhandene Mutationen vorab in vitro mit Methoden der

3 Auf Clinicaltrials.gov waren am 30.06.2020 insgesamt 57 Studien mit dem Schlagwort „Organoid“ gelistet, darunter befanden sich aber auch zahlreiche geplante Studien, die noch nicht mit der Rekrutierung von Teilnehmenden begonnen hatten. 31 Studien waren als rekrutierend angegeben und 6 als abgeschlossen, beendet oder zurückgezogen. Siehe unter: https://clinicaltrials.gov/ct2/resu lts?cond $=\&$ term $=$ organoid\&cntry $=\&$ state $=\&$ city $=\&$ dist $=[30.06 .2020]$. 
Genomeditierung korrigiert werden (siehe Teriyapirom/Batista-Rocha/Koo, Kap. 3.3). Eine Alternative wären allogene, also von anderen, gesunden Menschen abstammende, Organoidtransplantate, die vorher auf Immunkompatibilität getestet wurden. Im Vergleich von Organoiden aus adulten Stammzellen mit pluripotenten Stammzellen ist für die regenerative Medizin wichtig, dass Organoide aus adulten Stammzellen genetisch sehr stabil sind. Zudem entsteht hier nur ein Zelltyp (beispielsweise wie oben erwähnt nur epitheliale Zellen) und durch die eingeschränkte zelluläre Plastizität ist eine Entartung unwahrscheinlich. Organoide aus adulten Stammzellen einer Patientin oder eines Patienten sind untereinander sehr homogen, während Organoide aus pluripotenten Stammzellen einer Patientin oder eines Patienten heterogener sein können, wobei auch hier die Standardisierung schnell voranschreitet.

Organoide können auch für toxikologische Tests zur Bewertung der Toxizität und für Medikamentenscreenings in der Medikamentenentwicklung genutzt werden. Für diese Tests werden bisher meist Tiermodelle verwendet. Die Aussagekraft von Organoidtests im Vergleich zu Tierversuchen für die Wirkung von Stoffen wird derzeit erforscht. Das Potenzial von Organoiden, in Zukunft Tierversuche zu ergänzen, wird von manchen Forschenden aber als hoch eingeschätzt (siehe Interview mit Clevers, Kap. 2.2).

\subsubsection{Herausforderungen und Weiterentwicklung des Forschungsfeldes}

Gegenwärtige Herausforderungen der Organoidforschung bestehen in dem begrenzten Wissen über die biologischen Prozesse der Zelldifferenzierung und Organentstehung, beispielsweise der Zell-Zell-Interaktion und der Rolle von bestimmten Umgebungsfaktoren, die auch die Reproduzierbarkeit der Forschungsergebnisse beeinflussen. ${ }^{4}$ Eine wichtige technische Hürde ist hierbei der Einsatz der dreidimensionalen extrazellulären Matrix. Diese ist derzeit noch nicht vollständig chemisch definiert und kann noch nicht synthetisch hergestellt werden. Im Feld der pluripotenten Stammzellen ist der Prozess der Herstellung von Organoiden immer noch relativ zeit- und materialaufwendig, was die schnelle Herstellung großer Mengen an Organoiden z. B. für großangelegte Studien oder auch den medizinischen Einsatz schwierig macht. Es wird hier über automatisierte Prozesse nachgedacht (Czerniecki et al., 2018).

4 Unter Reproduzierbarkeit versteht man, dass bei gleichen experimentellen Bedingungen und Ausgangszellen zuverlässig einander sehr ähnliche Organoide entstehen, die sich in Merkmalen wie Größe, Form, Zellzusammensetzung und Struktur kaum unterscheiden (Huch et al., 2017). Da die Faktoren, die die Organoidentwicklung steuern, noch nicht vollständig bekannt und steuerbar sind, ist die Reproduzierbarkeit derzeit eingeschränkt. 
Ein weiteres Entwicklungsfeld ist die Erweiterung des zellulären Spektrums. Denn noch können nicht alle Organe in Form von Organoiden nachgebildet werden und die bereits entwickelten Organoide bilden nicht immer alle Zelltypen des Organs. Beispielsweise ist ein Darm umgeben von Blutgefäßen und Nerven, die aber im Organoid nicht enthalten sind. Dies schränkt die Übertragbarkeit von Forschungsergebnissen auf ein im Körper befindliches Organ erheblich ein. Großes Potenzial könnte die nächste Generation von Organoiden bieten, die komplexer und reifer sein und die Organe besser nachahmen können sollen, z. B. durch enthaltene Blutgefäße, Immunzellen oder Nervengewebe. Zur Erreichung von Komplexität für die Untersuchung von Wechselwirkungen zwischen Organen ist die Multi-Organ-on-a-Chip-Technologie von Bedeutung, bei der unterschiedliche Organoide auf einem Chip miteinander verknüpft werden können (z. B. Leber-, Nieren- und Lungenorganoide). Zudem können Organoide, beispielsweise Organoide verschiedener Hirnareale, zu sogenannten „Assembloiden“ zusammengesetzt werden. Auf diese Weise kann auch das Zusammenspiel verschiedener Zelltypen und Regionen bei Multiorganerkrankungen untersucht werden. Organoide dahingehend weiter zu entwickeln, dass sie bestimmten Organen möglichst ähnlich sind, ist mit Blick auf die genannten Zwecke ein wichtiges Forschungsziel. Wissenschaftsphilosophische Untersuchungen der Rolle von Modellen in der Organoidforschung zeigen jedoch, dass zwar Ähnlichkeiten zwischen Organoiden und Organen für die Absicherung von modellbasierten Schlussfolgerungen zentral sind, aber bestimmte Modellfunktionen gerade auch Unterschiede zwischen Organoiden und Organen voraussetzen, schließlich sind Modelle keine Kopien (siehe Fagan, Kap. 4). Dementsprechend erscheint das Ziel größtmöglicher Ähnlichkeit von Organoiden und Organen zwar wesentlichen, aber nicht allen Forschungszwecken angemessen (ebd.).

Eine wichtige technologische Weiterentwicklung der Organoidforschung vor allem zur Erforschung von Krankheiten stellt auch die gentechnische Veränderung von Organoiden oder vorangehenden Stammzellen durch unterschiedliche Methoden der Genomeditierung (z. B. CRISPR/Cas) dar (siehe Teriyapirom/Batista-Rocha/Koo, Kap. 3.3). Vielversprechend ist zudem der Einsatz von Verfahren der Live-Bildgebung und neuer Biomaterialien in der Organoidforschung. 


\subsubsection{Ethische Fragestellungen im Zusammenhang mit der Organoidforschung 5}

Durch die Organoidforschung und ihre Anwendungen werden zahlreiche ethische Fragen aufgeworfen, die zu einem großen Teil bereits in sie einbettenden Bereichen wie Forschung und Medizin im Allgemeinen oder der Stammzellforschung im Besonderen gestellt werden und sich z. B. auf Mensch-Tier-Chimären, die Forschung mit hES-Zellen, Genomeditierung, Tierversuche, Biobanken und Datenschutz oder Verteilungsgerechtigkeit beziehen (für einen Überblick zu bestehenden ethischen und damit verbundenen gesellschaftlichen, und rechtlichen Fragen siehe Tabelle 1 unter 2.1.7). Dabei werden viele dieser Fragen zwar erneut aufgegriffen, durch die voranschreitende Technologie einhergehend mit neuen Erkenntnissen, zunehmender Komplexität oder neuen Anwendungsmöglichkeiten, aber weiter verschärft.

Für die Organoidforschung spezifische und neue ethische Fragestellungen ergeben sich insbesondere für Hirnorganoide und Embryoide. Hirnorganoide werden aus humanen pluripotenten Stammzellen gewonnen und können unterschiedliche Regionen des menschlichen Gehirns in einem embryonalen oder frühen fetalen Stadium nachbilden (siehe hierzu Tanaka/Park, Kap. 3.5). In diesem Zusammenhang bestehen zwei ethische Fragenstränge, die die normative Bedeutung einerseits des menschlichen Gehirns auch bereits in frühen Embryonalstadien und andererseits der Bewusstseinsfähigkeit in deutlich späteren Entwicklungsstadien sowohl beim (geborenen) Menschen als auch bei vielen Tieren betreffen. Letzteres bezieht sich auf mögliche zukünftige, weiter gereifte und komplexere Hirnorganoide, Ersteres könnte sich bereits auf derzeitige Hirnorganoide beziehen. Im Zusammenhang mit dem Schwangerschaftsabbruch und dem ethischen und rechtlichen Status menschlicher Embryonen wird in Deutschland beispielsweise die Entwicklung der Gehirnfunktion (ab ca. dem 70. Tag nach der Befruchtung) als normatives Kriterium für einen vollen Lebensschutz diskutiert (Sass, 1989: 160 ff.). Auch international wird z. B. die sogenannte 14-Tage-Regel, die in vielen „Embryonenforschung“ zulassenden Ländern (wie z. B. USA, Kanada, Großbritannien) eine In-vitro-Entwicklung von menschlichen Embryonen über den 14. Tag hinaus verbietet, häufig damit begründet, dass das Auftreten des Primitivstreifens das erste Anzeichen eines sich ausbildenden Gehirns darstelle (Hostiuc et al., 2019: 119). Vor dem Hintergrund, dass die embryonale Hirnentwicklung damit in einigen Ländern bereits in frühen und frühesten Entwicklungsstadien als ethisches und rechtliches Schutzkriterium für den moralischen und rechtlichen Status menschlicher Em-

5 In Abschnitt 2.1.5 wird die ethische Analyse von Hannah Schickl skizziert, die nicht notwendigerweise die Auffassung sämtlicher Autorinnen widerspiegelt. 
bryonen angesehen wird, müsste die menschliche Hirnentwicklung konsistenterweise auch für Hirnorganoide herangezogen werden, was ein Forschungsverbot nach sich ziehen würde (ähnlich Taupitz, Kap. 7). Eine andere Möglichkeit wäre, die Hirnentwicklung als Schutzkriterium - zumindest für einen starken Status - aufzugeben zugunsten aktualer Fähigkeiten wie z. B. die Empfindungsfähigkeit, Bewusstseinsfähigkeit etc. Die Bewusstseinsfähigkeit ist zusammen mit der Empfindungsfähigkeit als moralisches Kriterium weithin anerkannt sowohl bezogen auf Menschen als auch innerhalb der Tierethikdebatte (vgl. z. B. Singer, 2013: $137 \mathrm{ff}$.). Entsprechend wird die Möglichkeit, dass (zukünftige) Hirnorganoide evtl. eine Art Bewusstsein ähnlich dem anderer Lebewesen entwickeln könnten, von manchen als bedenklich eingestuft. Es wurde in diesem Zusammenhang sogar angeregt, über eine rechtliche Vormundschaft für Hirnorganoide mit (menschlichen) mentalen Eigenschaften nachzudenken (Farahany et al., 2018). Problematisch ist dabei zum einen, dass unterschiedliche Eigenschaften und Fähigkeiten unter den Begriff der Bewusstseinsfähigkeit gezählt werden und diese zum anderen nicht absolut, sondern in Graden vorliegen. Aber selbst, wenn bestimmte Eigenschaften und Grade in ihrer jeweiligen normativen Relevanz identifiziert würden, bestünde immer noch ein methodisches Problem der Messbarkeit dieser Eigenschaften bei Hirnorganoiden. Von den meisten Autorinnen und Autoren (wie auch den Mitgliedern der IAG Gentechnologiebericht; siehe Kernaussagen und Handlungsempfehlungen) wird daher in Bezug auf eine mögliche Bewusstseinsfähigkeit zukünftiger Hirnorganoide momentan kein akuter Handlungsbedarf gesehen (siehe auch Taupitz, Kap. 7), sondern ein weitergehender wissenschaftlicher Forschungsbedarf begleitet von einem breiten gesellschaftlichen Diskurs zu diesem Thema gefordert (siehe auch Schicktanz, Kap. 6).

Embryoide werden ebenfalls aus humanen pluripotenten Stammzellen gewonnen und bilden Stadien der frühen Embryonalentwicklung ab, die sonst nur nach der Befruchtung (oder nach Einsatz alternativer Techniken wie beispielsweise dem somatischen Zellkerntransfer oder der Parthenogenese) in menschlichen Embryonen in vitro (Blastulation) oder in menschlichen Embryonen in vivo nach Implantation in einen Uterus (Gastrulation und Neurulation) in Erscheinung treten (Nicolas/Etoc/Brivanlou, Kap. 5). Der moralische Status menschlicher Embryonen in vitro und in vivo war und ist Bestandteil vieler ethischer Kontroversen, gleichzeitig räumen viele rechtliche Regelungen vor allem menschlichen Embryonen in vitro einen gewissen (z. B. 14-TageRegel) bis hohen (z. B. deutsches Embryonenschutzgesetz) Schutz ein. Da die Regelungen bzw. Verbote jeweils mit dem Begriff „menschlicher Embryo“ operieren - nach der Struktur: was ein menschlicher Embryo ist, wird so und so geschützt bzw. damit darf das und jenes nicht gemacht werden -, wird die internationale wissenschaftliche 
Debatte derzeit von der Frage dominiert, ob Embryoide Embryonen sind und die Regelungen insofern ebenso auf sie zutreffen oder nicht. Fast alle Autorinnen und Autoren verneinen die Frage und sind damit der Ansicht, dass bestehende Regelungen zum Embryonenschutz nicht auf sie zutreffen. Als Hauptargument wird dabei die mangelnde Entwicklungsfähigkeit von Embryoiden (bis zur Geburt) herangezogen. Vor dem Hintergrund immer weiter entwickelter Embryoide (vor allem im Mausmodell) ist diese Annahme allerdings ins Wanken geraten. Inzwischen unterscheiden einige Autorinnen und Autoren zwischen Embryoiden, die sich (theoretisch) zum Menschen entwickeln können, und Embryoiden, die das (aufgrund von fehlendem extra-embryonalen Gewebe) nicht können. Auf Grundlage der Richtlinien der internationalen Gesellschaft für Stammzellforschung (ISSCR) von 2016 wurden daher beispielweise spezifische neue Regelungen für Embryoide gefordert, die sich an bestehenden Regelungen zu menschlichen Embryonen orientieren sollen (vor allem an der 14-Tage-Regel, aber auch an anderen Verboten), sofern Embryoide über ein Entwicklungspotenzial zum Menschen verfügen (vgl. ISSCR, 2016; Hyun et al., 2020). Die normative Relevanz eines biologischen Entwicklungspotenzials ist allerdings ihrerseits ethisch hoch umstritten (Schickl et al., 2014). Stattdessen wird z. B. auf Basis der Empfindungsfähigkeit als normatives Kriterium gefordert, bestehende Regelungen zu menschlichen Embryonen grundsätzlich zu überdenken und die Forschung sowohl an Embryonen als auch an Embryoiden mit Entwicklungspotenzial über 14 Tage hinaus zuzulassen (siehe Nicolas/Etoc/Brivanlou, Kap. 5).

Insgesamt fällt auf, dass die wissenschaftliche ethische (und auch rechtliche) Debatte um Organoide in Deutschland noch in ihren zaghaften Anfängen steht, während international zumindest die angesprochenen Fragen zu bestimmten Organoiden diskutiert werden. Das mag auch daran liegen, dass die Forschung im naturwissenschaftlichen und medizinischen Bereich bisher vergleichsweise wenig nationale Förderung erfährt (siehe Osterheider/Koshelev/Reinschke/Marx-Stölting, Kap. 9) und in Deutschland aktuell auch noch kaum ELSA-Projekte durchgeführt werden.

\subsubsection{Organoidforschung: gesellschaftlicher Kontext und sozialwissenschaftliche Perspektiven}

Um dem komplexen Thema gerecht zu werden, sollte die Organoidforschung nicht isoliert, sondern eingebettet in ihren, gesellschaftlichen, ökonomischen und (forschungs-)politischen Kontext betrachtet werden. Dies wird allerdings dadurch erschwert, dass die Organoidforschung bislang nicht im Fokus sozial- und politikwissenschaftlicher Studien stand, was sich auch in der Zusammensetzung der Beiträge zu 
diesem Themenband widerspiegelt; außerhalb der Medizin und Naturwissenschaften werden im internationalen Diskurs bisher in erster Linie ethische und rechtliche Aspekte thematisiert. ${ }^{6}$ Jedoch lassen sich in Rückgriff auf die bereits bestehende vielfältige und breitgefächerte sozial-, politik- und kulturwissenschaftliche Literatur zur Stammzellforschung einige Probleme und Fragen skizzieren, die auch für die Forschung an Organoiden einschlägig sein dürften, da beide durch die Gewinnung von Organoiden aus Stammzellen materiell, institutionell, technologisch und sozioökonomisch miteinander verbunden sind.

Eine umfassende Darstellung ist an dieser Stelle nicht zu leisten, daher sollen exemplarisch einzelne für die Organoidforschung besonders wesentliche Themen und Ansätze genannt werden. Dazu gehören Analysen des bioökonomischen Kontextes, die es ermöglichen, Marktstrukturen, Interessen und Prozesse der ökonomischen (und ethischen) Inwertsetzung von Körperstoffen und ihre Rolle in der Entwicklung lebenswissenschaftlicher und biomedizinischer Forschung und Anwendung zu verstehen (Beltrame/Hauskeller, 2019; Sunder Rajan, 2012). Auch neue Bioobjekte (Vermeulen et al., 2012) wie z. B. die bereits erwähnten, in der Forschung verwendeten menschlichen Hirnorganoide in Mäusen oder anvisierte transplantierbare Organoide können durch einschlägige, empirisch fundierte, sozialwissenschaftliche Untersuchungen in ihrer gesellschaftlichen Bedingtheit und Bedeutung und in Hinblick auf die mit ihnen verbundenen Zukunftsvisionen erhellt werden. Einschlägige Studien sind von großem gesellschaftlichen Interesse, da sie zur intellektuellen und gesellschaftspolitischen Einordnung, angemessenen Regulierung sowie demokratischen Willensbildung über Formen und Grenzen der wissenschaftlichen, ökonomischen und medizinischen Nutzung entsprechender Bioobjekte beitragen können.

Insofern als ein wesentliches Ziel der Organoidforschung darin besteht, zur regenerativen Medizin ${ }^{7}$ beizutragen, sind sozial- und politikwissenschaftliche Studien der Bedingungen, Dynamiken und Entwicklungen regenerativer Medizin (z. B. Webster, 2013; Gottweis et al., 2009; Haddad et al., 2013) aufschlussreich für ein Verständnis der po-

6 Unsere Recherchen haben zwar viele naturwissenschaftliche und mediale sowie einige ethische Publikationen zutage gefördert, aber kaum sozialwissenschaftliche Beiträge (Stand Frühjahr 2020).

7 Regenerative Medizin unterscheidet sich insofern von herkömmlichen Ansätzen der Medizin, als sie darauf abzielt, durch die Verwendung neuer Biotechnologien die Funktionsfähigkeit von Zellen, Gewebe und Organen wiederherzustellen, zu erhalten bzw. zu verbessern durch die Stimulierung bzw. Erhöhung der körpereigenen Fähigkeit zur Selbstreparatur, wobei im Gegensatz zu herkömmlichen medikamentösen und biopharmazeutischen Ansätzen insbesondere die Veränderung der Zellstruktur und die Nutzung des regenerativen Potenzials von Stammzellen eine zentrale Rolle spielen (Webster, 2013:3). 
litischen, regulatorischen, epistemischen, institutionellen und gesellschaftlichen Herausforderungen der Organoidforschung und klinischen Translation organoidbasierter medizinischer Anwendungen in ihrem Zusammenhang. Auf Basis dessen lässt sich fragen, welche Formen und Kriterien für die Sicherheit und Wirksamkeit der klinischen Translation der Organoidforschung angemessen und welche Hürden absehbar sind. Im Zusammenhang damit sind einerseits die regulatorischen Herausforderungen des anvisierten Überganges organoid-basierter biomedizinischer Innovation in die Klinik vor dem Hintergrund vielfältiger Formen epistemischer Unsicherheit von Interesse, die bereits die klinische Translation der Stammzellforschung erschweren (Hauskeller 2018), andererseits sind die von Haddad (2019) in Bezug auf Stammzellen thematisierten Formen, Probleme und Herausforderungen post-pharmazeutischer Modelle der Gesundheitsforschung und -versorgung auch für Organoide relevant.

Fortschritte auf dem Gebiet der regenerativen Medizin und große gesellschaftliche Hoffnungen haben Stimmen laut werden lassen, die eine moralische Verpflichtung postulieren, translationale Forschung zu beschleunigen (Feidt et al., 2019). Demgegenüber warnen Kritikerinnen und Kritiker vor möglichen Risiken für die Patientensicherheit und Forschungsqualität durch die Beschleunigung des Übergangs von der Laborforschung in die klinische Praxis bzw. einer zu starken Ausrichtung Ersterer auf Letztere (vgl. Maienschein et al., 2008). Diese insbesondere im Kontext der Stammzellforschung geführten Kontroversen sind für die Forschung an Organoiden lehrreich, wobei sich aufgrund der größeren Komplexität von Organoiden Fragen nach Kriterien hinreichender wissenschaftlicher Evidenz für ausreichende Effizienz und Patientensicherheit sowie der Validität von Forschungsergebnissen noch einmal verschärfen. Die Anwendbarkeit etablierter Verfahren randomisierter Kontrollstudien wurde beispielsweise bei anvisierten Transplantationen von Stammzellen diskutiert aufgrund der teilweise schwierigen Standardisierbarkeit. Auch bei organoidbasierten medizinischen Anwendungen sollten angemessene Kriterien der Wirksamkeit und Sicherheit untersucht werden und hier die inhärenten Unterschiede der beiden Organoidtechnologien insbesondere in der Heterogenität verglichen werden. ${ }^{8}$

Grundsätzlich ist festzuhalten, dass der Zugang zu den Lebenswissenschaften, insbesondere die Konzeption des Verhältnisses von Wissenschaft und Gesellschaft in

8 Organoide aus adulten Stammzellen unterscheiden sich vor allem zwischen den Patientinnen und Patienten; Organoide eines einzelnen Patienten bzw. einer einzelnen Patientin hingegen sind homogen. Heterogen auf beiden genannten Ebenen sind Organoide aus pluripotenten Stammzellen. Letztere werden aber oft aus hochstandardisierten und gut charakterisierten hiPS- oder hES-Zell-Linien abgeleitet: so wird die Heterogenität zwischen den Patientinnen und Patienten aufgefangen. Dies wäre vor allem für eine allogene Transplantation interessant. 
den genannten Untersuchungen von herkömmlichen, im Selbstverständnis vieler Naturwissenschaftler/-innen, der Öffentlichkeit und auch ethischen Analysen vorherrschenden Auffassungen unterschieden ist, die häufig nach wie vor Wissenschaft und Gesellschaft als getrennte Bereiche konzipieren. Viele sozialwissenschaftliche Studien hingegen, insbesondere aus den Science and Technology Studies, erforschen die gesellschaftlichen, ökonomischen, politischen, kulturellen und ideologischen Bedingungen und Prozesse, die lebenswissenschaftliche Forschung und Anwendung ermöglichen und formen und wiederum von Wissenschaft und Technologie geprägt und verändert werden. Das wechselseitig bedingte Entstehen und Entwickeln wissenschaftlicher und gesellschaftlicher Phänomene und Praktiken ist auf den Begriff „,co-production“ (Jasanoff, 2004) gebracht worden. Dieses Konzept wurde am Beispiel der Stammzellforschung vor Kurzem weiter entwickelt zu dem multidisziplinären Matrix-Zugang, der der Verwobenheit epistemischer, forschungspragmatischer, gesellschaftlicher, ethischer und politischer Dimensionen wissenschaftlich-technischer Entwicklungen Rechnung trägt und erhellt, inwiefern auch die Geistes- und Sozialwissenschaften in ihrer Auseinandersetzung mit den Lebenswissenschaften deren Gestalt und Entwicklung mit beeinflussen (Hauskeller et al., 2019). Entsprechende Analysen der Organoidforschung sind ein Desiderat.

Kommunikationswissenschaftliche Studien, wie z. B. Befragungen, experimentelle Studien oder auch Diskursanalysen, die die Wahrnehmung von Organoiden oder auch die Einstellungen zur Organoidforschung in der breiteren Bevölkerung in den Blick nehmen, gibt es bisher nicht. Bereits durchgeführte Untersuchungen (allgemein zur Humangenomforschung: Gerhards/Schäfer, 2006; zur Biomedizin: Peters et al., 2008; zur Stammzellforschung: Nisbet, 2005; Ho et al., 2008; Liu/Priest, 2009; Peddie et al., 2009; Bates et al., 2010; Critchley, 2013; Shih et al., 2013) zur Wahrnehmung der Stammzellforschung und der Kommunikation darüber deuten jedoch darauf hin, dass interessante Befunde möglich sind; gerade vor dem Hintergrund des anhaltend kontrovers diskutierten Einsatzes von hES-Zellen sowohl in der Öffentlichkeit als auch unter Forschenden. So ist die Wahrnehmung von Organoiden seitens der Öffentlichkeit, aber auch die Analyse der Medienberichterstattung zu diesem Themenfeld ein interessanter Aspekt, der sich zu erforschen lohnt. Erste Hinweise darauf gibt eine Analyse der Medienberichterstattung hinsichtlich der Thematisierung von Organoiden (Süddeutsche Zeitung [SZ], Frankfurter Allgemeine Zeitung [FAZ], Der Spiegel, Die Zeit; Untersuchungszeitraum: 01.12.2013 bis zum 01.12.2019) (siehe Osterheider/Koshelev/Reinschke/Marx-Stölting, Kap. 9). Vor allem die Forschung auf dem Gebiet der Hirnorganoide und der Embryoide sowie das ontologische Verständnis, die ethische 
Beurteilung und der rechtliche Status standen im Mittelpunkt der Medienberichterstattung.

\subsection{7 Überblick über ethische, rechtliche und gesellschaftliche Fragestellungen im Zusammenhang mit Organoiden in Forschung und Anwendung ${ }^{9}$}

Eine exemplarische, stichwortartige Zusammenschau der ethischen, gesellschaftlichen sowie rechtlichen Fragen zu Herstellung und Verwendung von Organoiden in Forschung und medizinischer Anwendung bietet Tabelle 1.

Tabelle 1: Ethische, rechtliche und gesellschaftliche Fragen zur Organoidforschung

\begin{tabular}{|c|c|}
\hline Thema & Offene Fragen und Probleme \\
\hline Hirnorganoide & $\begin{array}{l}\text { - Sollte Hirnorganoiden derselbe ethische und rechtliche Status wie menschlichen Embryo- } \\
\text { - } \quad \text { nen zugeschrieben werden? } \\
\text { - } \quad \text { methodisches Verständnis von Bewusstsein und Personalität } \\
\text { - Bedarf es spezifischer Eer Messbarkeit mentaler und kognitiver Prozesse } \\
\text { - Transplantation in Versuchstiere? } \\
\quad \text { gollten Zellspenderinnen und -spender ihre Zustimmung geben zur Herstellung von Hirnor- } \\
\text { ganoiden aus ihren Zellen? } \\
\text { rowishittsenschaften: Hirntoddebatte, Tierethik, Embryonenethik, Philosophie des Geistes und Neu- }\end{array}$ \\
\hline $\begin{array}{l}\text { Mensch-Tier-Chi- } \\
\text { mären }\end{array}$ & $\begin{array}{l}\text { - } \quad \text { moralische Bewertung von Speziesgrenzen } \\
\text { - ontologisches Verständnis, moralischer und rechtlicher Status der entstandenen Entitäten } \\
\text { - } \quad \text { (insbesondere in Bezug auf Tiere, in die menschliche Hirnorganoide transplantiert werden) } \\
\text { - Schnittstellen: Diskussion um Xenotransplantation und Tierversuche }\end{array}$ \\
\hline
\end{tabular}

9 Einen Überblick über ethische, rechtliche und gesellschaftliche Fragen zur Organoidforschung bieten insbesondere: Aach et al., 2017; Boers et al., 2016; Bredenoord et al., 2017; Lavazza/Massimini, 2015; Munsie et al., 2015; Shepherd, 2018. 


\begin{tabular}{|c|c|}
\hline $\begin{array}{l}\text { Forschung mit } \\
\text { Embryonen, hES- } \\
\text { Zellen, fetalen } \\
\text { Zellen und Em- } \\
\text { bryoiden }\end{array}$ & $\begin{array}{l}\text { - Dürfen Zellen und Gewebe von (toten, u. U. abgetriebenen) Feten für die Forschung } \\
\text { verwendet werden? } \\
\text { - } \quad \text { moralischer Status von menschlichen Embryonen, hES-Zellen und Embryoiden } \\
\text { - } \quad \text { ontologischer Status von Embryoiden: Sind sie menschliche Embryonen? } \\
\text { wicklungsfähigkeit und deren normatives Gewicht. Sind bestehende rechtliche Regelungen } \\
\text { - zu menschlichen Embryonen auf Embryoide anzuwenden? } \\
\text { - Bedarf es spezifischer Ethikkommissionen für die Herstellung von Embryoiden? } \\
\text { Müssen Zellspenderinnen und -spender ihre Zustimmung geben zur Herstellung von Em- } \\
\text { bryoiden aus ihren Zellen? } \\
\text { Schnittstellen: Stammzellforschung, Embryonenforschung, Abtreibungsdebatte und Embryo- } \\
\text { nenethik }\end{array}$ \\
\hline Tierversuche & $\begin{array}{l}\text { - Auswirkungen der Organoidforschung: Beitrag zum Ziel der 3R („replacement, refinement, } \\
\text { - } \quad \text { Umsetion“) oder mehr Tierversuche als Folge? } \\
\text { - Schutz der für Versuche und andere wissenschaftliche Zwecke verwendeten Tiere” } \\
\text { - Tierschutz als Staatsziel in deutscher Verfassung verankert } \\
\text { - Schnittstelle: Tierethik }\end{array}$ \\
\hline $\begin{array}{l}\text { Konvergen } \\
\text { medizinisc } \\
\text { Technolog } \\
\text { Beispiel Ger }\end{array}$ & $\begin{array}{l}\text { - Konvergenz von z. B. Stammzellforschung, anderen Gentechnologien, Transplantationsme- } \\
\text { dizin, Präzisionsmedizin mit Organoidforschung } \\
\text { Die Konvergenz ist verbunden mit einem Geflecht aus Regulierungen, ethischen Debatten } \\
\text { und schwer einschätzbaren Risiken } \\
\text { Schnittstelle: Genomeditierung und damit verbundene ethische Fragen der Anwendung am } \\
\text { Menschen und an Tieren }\end{array}$ \\
\hline $\begin{array}{l}\text { Nut- } \\
\text { an } \\
\text { or- } \\
\text { en }\end{array}$ & $\begin{array}{l}\text { - } \quad \text { Biobanken und geeignete Formen der Einwilligung (z. B. Broad oder Dynamic Consent) in } \\
\text { die Lagerung bzw. Speicherung und Nutzung von Biomaterialien und Daten } \\
\text { - } \quad \text { Datensicherheit } \\
\text { - } \quad \text { nen und Probanden oder Patientengruppen? } \\
\text { - Kommodifizierung des menschlichen Körpers: Verändertes Verständnis und Verhältnis zum } \\
\text { - } \quad \text { Pagenen Körper und dem anderer? „Ersatzteillager Mensch“? } \\
\text { - Schnittstellen: Stammzellforschung, Tissue Engineering, Transplantationsmedizin, Hirntod } \\
\text { und Organtransplantation, Biopatente }\end{array}$ \\
\hline & $\begin{array}{l}\text { - Allokation von Forschungsgeldern: Was wird erforscht und was nicht? Wessen Bedürfnisse } \\
\text { und Interessen werden dabei berücksichtigt? } \\
\text { - Finanzierung von und Zugang zu kostenintensiven Therapien } \\
\text { - bioökonomischer Kontext, Biotech-Industrie: Wer macht Gewinn unter welchen Bedingun- } \\
\text { gen, mit welchen Konsequenzen? Wie wirkt sich dies auf die Forschung und Entwicklung } \\
\text { von Therapien aus? } \\
\text { - Schnittstellen: Forschungsstandort Deutschland, Infrastrukturen, Braindrain }\end{array}$ \\
\hline
\end{tabular}




\begin{tabular}{|c|c|}
\hline $\begin{array}{l}\text { Translation in die } \\
\text { Klinik }\end{array}$ & $\begin{array}{l}\text { - Anwendbarkeit von (umstrittenen Standards) für Effizienz und Sicherheit in Klinischen } \\
\text { Prüfungen - geeignete Kriterien? } \\
\text { - Grenzen der Vergleichbarkeit von Organoiden mit Organen im Körper/Vermeidbarkeit von } \\
\text { Abstoßungsreaktionen } \\
\text { - Rolle der Biotechindustrie: Was wird (nicht) erforscht, beispielsweise durch Ausrichtung auf } \\
\text { die Herstellung von Blockbustern? } \\
\text { Voraussetzungen und Rahmenbedingungen für Forschung in Unternehmen und akademi- } \\
\text { sche klinische Versuche (Probleme für Ansätze regenerativer Medizin durch Ausrichtung } \\
\text { der Medikamentenentwicklung und Regulierung klinischer Versuche auf standardisiert } \\
\text { anwendbare Medikamente) } \\
\text { Schnittstellen: klinische Forschung, regenerative Medizin }\end{array}$ \\
\hline Validitätsfragen & $\begin{array}{l}\text { - Szenarien klinischer Anwendung und deren Verhältnis zum gegenwärtigen Wissensstand, } \\
\text { Methoden und Problembewusstsein } \\
\text { - Übertragbarkeit von Erkenntnissen der Organoidforschung auf den lebenden (gesunden } \\
\text { oder erkrankten) Organismus } \\
\text { - Verlässlichkeit von organoidbasierten Toxizitätsstudien (evtl. im Vergleich mit In-vivo-Tier- } \\
\text { versuchen) }\end{array}$ \\
\hline Sprache & $\begin{array}{l}\text { - Begriffe und Bezeichnungen (z. B. "Organoid“, „Embryoid“; , "Selbstorganisation”, ", Toti- } \\
\text { potenz" etc.) werden uneinheitlich verwendet (unterschiedliche Definitionen eines Begriffs } \\
\text { und unterschiedliche Bezeichnungen einer Entität) und sind zum Teil normativ aufgeladen } \\
\text { - Metaphernanalyse, Diskurskontexte (die Bezeichnungen „Organoide” oder "Embryoide” } \\
\text { suggerieren eine große Ähnlichkeit bei gleichzeitiger Betonung der Nicht-Gleichheit) }\end{array}$ \\
\hline $\begin{array}{l}\text { Wissenschafts- } \\
\text { kommunikation }\end{array}$ & $\begin{array}{l}\text { - Wie werden Organoide und die Organoidforschung in der Öffentlichkeit wahrgenommen? } \\
\text { - } \quad \text { Medialisierung der Wissenschaft } \\
\text { - } \quad \text { zerständliche) Darstellung von Inhalten und Risiken } \\
\text { - Vertrauen in Kommunikatoren (Forschende, Wissenschaftseinrichtungen, Verbände/Verei- } \\
\text { ne, Unternehmen) und ihre verwendeten Kommunikationskanäle und Inhalte }\end{array}$ \\
\hline Umgang mit Hype & $\begin{array}{l}\text { - Umgang mit „Hype-and-Hope“-Technologien } \\
\text { - Umgang mit großen Hoffnungen von Patientinnen und Patienten } \\
\text { - } \quad \text { seriöse Kommunikation von Potenzialen und Risiken } \\
\text { - } \quad \text { Vedizinische Risiken möglicher verfüühter Anwendungen } \\
\text { - Könnte es zu einer Art "Organoidtourismus" kommen, wenn es bestimmte Therapieange- } \\
\quad \text { bote in anderen Ländern, aber in Deutschland nicht gibt? } \\
\text { - Schnittstelle: Wissenschaftskommunikation }\end{array}$ \\
\hline $\begin{array}{l}\text { Kultureller und } \\
\text { sozioökonomi- } \\
\text { scher Kontext }\end{array}$ & $\begin{array}{l}\text { - möglicherweise unterschiedliche ethische Bewertung und Wahrnehmung von Organoiden } \\
\text { und Chimären in verschiedenen Kulturen } \\
\text { - sozioökonomische Bedingungen und globale Regulierung } \\
\text { - mögliche gesellschaftliche/ökonomische Probleme in nicht-westlichen Gesellschaften, Ver- } \\
\text { ständnis von Organoiden }\end{array}$ \\
\hline
\end{tabular}

\subsubsection{Zum Inhalt dieses Themenbands}

Das diese Einleitung abschließende, von Sina Bartfeld geführte Interview mit Hans Clevers bietet einen anschaulichen Einstieg in die Thematik. Aus Sicht eines der 
führenden Forscher im Bereich der Organoidtechnologie werden darin sowohl die Anfänge der Organoidforschung als auch gegenwärtige und künftige Entwicklungen dargestellt. Darüber hinaus betont Hans Clevers die Notwendigkeit, ethische und rechtliche Expertise in die gesellschaftliche Deliberation über Möglichkeiten und Grenzen der Forschung an und Nutzung von Organoiden einzubinden und gibt einen Einblick in sein ganz persönliches moralisches Empfinden angesichts von Organoiden, die das menschliche Gehirn, den menschlichen Embryo oder den weiblichen Uterus nachbilden.

Im Anschluss an das Interview bietet der erste Teil des Themenbands einen Überblick über den naturwissenschaftlichen Forschungsstand. Basierend auf den englischen Originalbeiträgen aus einem parallel von der IAG Gentechnologiebericht veröffentlichten Special Issue (SI) im Journal of Molecular Medicine ${ }^{10}$ werden aktuelle wissenschaftliche Entwicklungen unterschiedlicher Bereiche der Organoidforschung kurz und allgemeinverständlich zusammengefasst (von Lilian Marx-Stölting und Anja Pichl). Damit erhalten alle, die nicht in diesem Spezialgebiet der Stammzellforschung tätig sind, einen deutschsprachigen Einblick in das vielfältige Forschungsgeschehen der Gegenwart. Die Reviews behandeln die Bedeutung von Organoiden in der Entwicklungsbiologie (Frum/Spence, Kap. 3.1), die Selbstorganisation von Organoiden aus Entodermzellen (Lewis/Keshara/Kim/Grapin-Botton, Kap. 3.2), das Genetic Engineering von Organoiden (Teriyapirom/Batista-Rocha/Koo, Kap. 3.3), Organoide in der Krebsforschung (Kretzschmar, Kap. 3.4), Hirnorganoide (Tanaka/Park, Kap. 3.5), Nierenorganoide (Gupta/Dilmen/Morizane, Kap. 3.6), Organoide des weiblichen Reproduktionstrakts (Chumduri/Turco, Kap. 3.7) und Organoide als Modell der angeborenen Immunität des Epithels im Magen-Darm-Trakt (Kayisoglu/Schlegel/Bartfeld, Kap. 3.8).

Der zweite Teil des Buches beschäftigt sich mit epistemischen, ethischen, rechtlichen, und gesellschaftlichen Fragen und Implikationen der Forschung an und potenziellen Anwendungen von Organoiden. Zu Beginn werden konzeptuelle sowie methodologische Fragen der Organoidforschung aus der Perspektive der Wissenschaftsphilosophie diskutiert. Da naturwissenschaftliche und medizinische Forschung häufig durch einen pragmatischen Umgang mit Gegenstandskonzepten gekennzeichnet ist und der öffentliche sowie ethische, rechtliche und sozialwissenschaftliche Diskurs sich auf diese Konzepte bezieht, ist eine philosophische Klärung der konzeptuellen Grundlagen der wissenschaftlichen Forschung von Bedeutung. Insofern verbindet der Beitrag der

10 Das Special Issue „3D Organoids“ wurde von der IAG unter Leitung von Sina Bartfeld zusammen mit Bon-Kyong Koo (Wien) und Cantas Alev (Kyoto) herausgegeben und ebenso wie dieser Band 2020 im Open Access veröffentlicht. 
Wissenschaftsphilosophin Melinda Bonnie Fagan den naturwissenschaftlichen Teil des Bandes mit dem ethisch/rechts- und sozialwissenschaftlichen Teil. Sie untersucht auf Basis philosophischer Erkenntnisse über wissenschaftliche Modelle und Modellierung die Funktion von Organoiden in der naturwissenschaftlichen Forschung (Kap. 4). Paola Nicolas, Fred Etoc und Ali H. Brivanlou bieten einen Überblick zur derzeitigen Forschung an Embryoiden und entwickeln Kriterien für einen zukünftigen konsistenten ethischen und rechtlichen Umgang mit synthetischen und natürlichen Embryonen (Kap. 5). Im Anschluss gibt Silke Schicktanz - den naturwissenschaftlichen Beitrag von Yoshiaki Tanaka und In-Hyun Park mit einer ethischen Diskussion komplementierend (Kap. 3.5) - einen Überblick zu forschungsethischen Fragen in der wissenschaftlichen Debatte um Hirnorganoide und Mensch-Tier-Chimären in der Organoidforschung (Kap. 6). Jochen Taupitz und Fruzsina Molnár-Gábor stellen die deutsche Rechtslage zu Organoiden im Allgemeinen (Taupitz, Kap. 7) sowie zu speziellen Fragen des internationalen Datenschutzes und der Einwilligung im Kontext von Organoidbiobanken (Molnár-Gábor, Kap. 8) umfassend dar.

Der Themenband wird auf bewährte Art und Weise mit einer Problemfeldanalyse und Indikatorenerhebung abgeschlossen (Osterheider/Koshelev/Reinschke/MarxStölting, Kap. 9). Basierend auf einer qualitativen Auswertung der Medienberichterstattung über Organoide wurden Problemfelder erhoben. Diese werden verstanden als Themenbereiche und Fragestellungen, die von der Öffentlichkeit wahrgenommen oder mitunter kontrovers diskutiert werden. Illustriert werden diese Problemfelder anhand quantitativer Daten (Indikatoren). Drei Themen respektive Problemfelder stehen im Fokus der Berichterstattung: der Status von Organoiden, ethische Implikationen und die Realisierung medizinischer Zielsetzungen. Hierbei ist bemerkenswert, dass im Untersuchungszeitraum Forschung auf dem Gebiet der Hirnorganoide und Embryoide im Mittelpunkt steht.

Angesichts der vielen drängenden, potenziell kontroversen und dabei noch offenen naturwissenschaftlichen, medizinischen, wissenschaftstheoretischen, kommunikationswissenschaftlichen, ethischen und rechtlichen Fragen, ist eine Untersuchung des Themenbereichs aus verschiedenen wissenschaftlichen Perspektiven erforderlich. Dieser Themenband will hierzu einen ersten Beitrag leisten und einen Anstoß für eine breite wissenschaftliche und gesellschaftliche Debatte liefern.

\subsubsection{Literaturverzeichnis}

Aach, J. et al. (2017): Addressing the ethical issues raised by synthetic human entities with embryolike features. In: eLife 6, Online-Publikation 21.03.2017. DOI: 10.7554/eLife.20674. 
Bartfeld, S./Clevers, H. (2018): Aus Stammzellen abgeleitete Organoide und ihre Bedeutung für die biomedizinische Forschung und Therapie. In: Zenke, M. et al. (Hrsg.): Stammzellforschung. Aktuelle wissenschaftliche und gesellschaftliche Entwicklungen. Nomos, Baden-Baden: 90-95.

Bates, S. R. et al. (2010): 'How do we know it's not been done yet?!' Trust, trust building and regulation in stem cell research. In: Science and Public Policy 37(9): 703-718. DOI: 10.1093/spp/ 37.9.703.

Beltrame, L./Hauskeller C. (2019): Assets, Commodities and Biosocialities. Multiple Biovalues in Hybrid Biobanking Practices. In: Tecnoscienza: Italian Journal of Science \& Technology Studies 9 (2): 5-31.

Boers, S. N. et al. (2016): Organoid biobanking: Identifying the ethics. Organoids revive old and raise new ethical challenges for basic research and therapeutic use. In: EMBO Reports 17(7): 938-941.

Bredenoord, A. L. et al. (2017): Human tissues in a dish: The research and ethical implications of organoid technology. In: Science 355(6322), Online-Publikation 20.02.2017. DOI: 10.1126/science.aaf9414.

Clevers, H. (2020): COVID-19: organoids go viral. In: Nature Reviews Molecular Cell Biology 21: 355356.

Critchley, C. R. et al. (2013): The impact of commercialisation on public perceptions of stem cell research: exploring differences across the use of induced pluripotent cells, human and animal embryos. In: Stem Cell Reviews and Reports 9(5): 541-554. DOI: 10.1007/s12015-013-9445-4.

Czerniecki, S. M. et al. (2018): High-throughput screening enhances kidney organoid differentiation from human pluripotent stem cells and enables automated multidimensional phenotyping. In: Cell Stem Cell 22: 929-940. DOI: 10.1016/j.stem.2018.04.022.

Farahany, N. A. (2018): The ethics of experimenting with human brain tissue. In: Nature 556: 429-432. Feidt, R. H. et al. (2019): A moral obligation to accelerating translational research: the "translational imperative". In: Sci Eng Ethics 25(1): 33-52.

Gerhards, J./Schäfer, M. S. (2006): Die Herstellung einer öffentlichen Hegemonie: Humangenomforschung in der deutschen und US-amerikanischen Presse. VS Verlag für Sozialwissenschaften, Wiesbaden.

Gottweis, H. et al. (2009): The global politics of human embryonic stem cell science. Regenerative medicine in transition. Palgrave Macmillan, Basingstoke.

Haddad, C. et al. (2013): Unruly objects: novel innovation paths and their regulatory challenge. In: Webster, A. (Hrsg.): The global dynamics od regenerative medicine. A social science critique. Palgrave Macmillan, Basingstoke: 88-117.

Haddad, C. (2019): Embodied values: Post-pharmaceutical health and the accumulation of surplus vitality in regenerative stem cell medicine. In: Sociologias, Porto Alegre, ano 21, n. 50: 48-79. DOI: 10.1590/15174522-02105002.

Hauskeller, C. (2018): Between the local and the global: Evaluating European regulation of stem cell regenerative medicine. In: Perspect Biol Med, 61(1): 42-58.

Hauskeller, C. et al. (2019): Knowledge and normativity. A matrix of disciplines and practices. In: Hauskeller, C. et al. (Hrsg.): The matrix of stem cell research. An approach to rethinking science in society. Routledge, London. 
Ho, S. S. et al. (2008): Effects of value predispositions, mass media use, and knowledge on public attitudes toward embryonic stem cell research. In: International Journal of Public Opinion Research 20(2): 171-192. DOI: 10.1093/ijpor/edn017.

Hostiuc, S. et al. (2019): The moral status of cerebral organoids. In: Regenerative Therapy 10: 118-122.

Huch, M. et al. (2017): The hope and the hype of organoid research. In: Development 144: 938-941. DOI: $10.1242 / \operatorname{dev} .150201$.

Hyun, I. et al. (2020): Toward guidelines for research on human embryo models formed from stem cells. In: Stem Cell Reports 14(2): 169-174.

ISSCR (2016): Guidelines for stem cell research and clinical translation. Unter: https://www.isscr.org /docs/default-source/all-isscr-guidelines/guidelines-2016/isscr-guidelines-for-stem-cell-research -and-clinical-translationd67119731dff6ddbb37cff0000940c19.pdf?sfvrsn=4 [13.05.2020].

Jasanoff, S. (2004): The idiom of co-production. In: Jasanoff, S. (Hrsg.): States of knowledge. The co-production of science and social order. Routledge, London: 1-12.

Lavazza, A./Massimini, M. (2018): Cerebral organoids: ethical issues and consciousness assessment. In: Journal of Medical Ethics 44(9): 606-610.

Liu, H./Priest, S. (2009): Understanding public support for stem cell research: media communication, interpersonal communication and trust in key actors. In: Public Understand. Sci. 18(6): 704-718. DOI: $10.1177 / 0963662508097625$.

Maienschein, J. et al. (2008): The ethos and ethics of translational research. In: The American Journal of Bioethics 8(3): 43-51.

Mallapaty, S. (2020): Mini-organs reveal how the coronavirus ravages the body. In: Nature 585: 15-16.

Munsie, M. et al. (2015): Ethical issues in human organoid and gastruloid research. In: Development 2017(144): 942-945.

Nisbet, M. C. (2005): The competition for worldviews: Values, information, and public support for stem cell research. In: International Journal of Public Opinion Research 17(1): 90-112. DOI: 10.1093/ijpor/edh058.

Peddie, V. L. et al. (2009): 'Not taken in by media hype': how potential donors, recipients and members of the general public perceive stem cell research. In: Human Reproduction 24(5): 11061113. DOI: $10.1093 /$ humrep/den496.

Peters, H. P. et al. (2008): Das Verhältnis von Wissenschaft und Massenmedien und die politische Relevanz öffentlicher Kommunikation über Wissenschaft am Beispiel der Biomedizin. Unter: http:// www.hpp-online.de/downloads/Peters_et_al_2009a.pdf [20.08.2020].

Sass, H. M. (1989): Hirntod und Hirnleben. In: Sass, H. M. (Hrsg.): Medizin und Ethik. Reclam, Stuttgart: $160-183$.

Schickl, H. et al. (2014): Abweg Totipotenz. Rechtsethische und rechtspolitische Herausforderungen im Umgang mit induzierten pluripotenten Stammzellen. In: Medizinrecht 32: 857-862. DOI: 10.1007/s00350-014-3863-4. 
Shepherd, J. (2018): Ethical (and epistemological) issues regarding consciousness in cerebral organoids. In: Journal of Medical Ethics 44(9): 611-612.

Shih, T.-J. et al. (2013): Disagreement and Value Predispositions: Understanding Public Opinion About Stem Cell Research. In: International Journal of Public Opinion Research 25 (3): 357-367. DOI: 10.1093/ijpor/eds029.

Singer, P. (2013): Praktische Ethik. 3. Aufl. Stuttgart, Reclam.

Sunder Rajan, K. (2012): Introduction: the capitalization of life and the liveliness of capital. In: Sunder Rajan, K. (Hrsg.): Lively capital. Biotechnology, ethics and governance in global markets. Duke University Press, Durham/London.

Vermeulen, N. et al. (Hrsg.) (2012): Bio-objects: life in the 21st century. Ashgate, London.

Webster, A. (2013): Introduction. The boundaries and mobilities of regenerative medicine. In: Webster, A. (Hrsg.): The global dynamics od regenerative medicine. A social science critique. Palgrave Macmillan, Basingstoke. 


\section{Sina Bartfeld}

\subsection{Das Potenzial von Organoiden realisieren ${ }^{11}$}

Ein Interview mit Hans Clevers

Aus dem Englischen übersetzt von Rike Zietlow

Seit einigen Jahren erregt ein neues, vielversprechendes Modell zur Erforschung menschlicher Krankheiten immer mehr Aufmerksamkeit: Organoide. Darunter versteht man aus humanen Stammzellen gezüchtete, dreidimensionale Gebilde, die vom Aufbau her einem Organ im Miniaturformat ähneln. Prinzipiell können solche Organoide aus Zellen eines jeden Patienten gezüchtet werden - womit sich vollkommen neue Möglichkeiten für die medizinische Forschung eröffnen. Dazu gehören personalisierte Therapien, Toxizitätstests und die Medikamentenentwicklung. Mittlerweile wird man sich allerdings sowohl in der Wissenschaft als auch in der Öffentlichkeit zunehmend der ethischen Implikationen bewusst: Denn solche Organoide können mittlerweile immer mehr Gewebearten nachbilden, zum Beispiel das Gehirn, den weiblichen Reproduktionstrakt und embryonale Frühstadien, während sich parallel dazu auch die Technologien für das sogenannte Gene-Editing immer weiter entwickeln. Eine der treibenden Kräfte der Organoidforschung ist Hans Clevers vom Hubrecht Institut in Utrecht in den Niederlanden. In diesem Interview diskutiert er das Potenzial der Organoidtechnologie für die Medizin, den aktuellen Stand der Forschung und die ethischen Implikationen. Das Interview führt Sina Bartfeld, eine ehemalige Mitarbeiterin des Clevers-Labors, die inzwischen eine unabhängige Forschungsgruppe an der Julius-Maximilians-Universität Würzburg leitet.

SB: Die Entwicklung von Organoiden ist unglaublich schnell vorangeschritten, zwischen ihrer Entdeckung und dem ersten Einsatz in der Klinik lagen nicht einmal zehn Jahre. Was war im Rückblick der erste Durchbruch?

HC: Zunächst halte ich es für wichtig, zwischen zwei verschiedenen Technologien zu unterscheiden, weil dafür zwei ganz unterschiedliche Arten von Stammzellen verwendet werden. Einerseits gibt es die sogenannten pluripotenten Stammzellen, aus

11 Dieser Beitrag ist eine Übersetzung des Editorials im Special Issue „3D Organoids“, das $2020 \mathrm{im}$ Journal of Molecular Medicine erschienen ist. 
denen sich der gesamte Organismus entwickeln kann. Dies sind entweder induzierte pluripotente Stammzellen oder embryonale Stammzellen, kurz iPS-Zellen oder ES-Zellen. Vielen Leuten ist jedoch nicht bewusst, dass es noch eine zweite Art von Stammzellen gibt. Diese sogenannten adulten Stammzellen regenerieren lebenslang unsere Gewebe. Zum Beispiel wird durch die adulten Stammzellen des Darms die aus Epithelzellen bestehende Darmschleimhaut fortlaufend ersetzt. Beide Forschungsgebiete, die iPS-/ES-Zell-Forschung und die adulte Stammzellforschung, haben jeweils ihre eigene Organoidtechnologie entwickelt.

SB: Und beide Forschungsfelder hatten ihre Durchbrüche.

HC: Ich denke, der Durchbruch für die iPS-/ES-Zell-Forschung war die Etablierung der ersten Organoide durch Yoshiki Sasai. ${ }^{12}$ Davor wollten Wissenschaftler, die an ESund iPS-Zellen forschten, daraus immer nur Zellen einer bestimmten Art generieren, etwa noch mehr Stammzellen, Beta-Zellen, Leberzellen oder Neuronen. Sasai bemerkte als erster, dass man stattdessen sogar richtige Strukturen herstellen kann, indem man den pluripotenten Stammzellen vor der Differenzierung erlaubte, Zellklumpen zu bilden. Sasai generierte damals kortikale Neuronen aus diesen undifferenzierten Zellklumpen und bemerkte, dass sie sich strukturiert organisierten. Er benutzte noch nicht den Begriff „Organoide“, aber er beschrieb bereits, dass die entstandenen Gebilde der Anatomie eines Organs ähneln. Dies war der Startschuss für die ES-/iPS-zellbasierte Organoidforschung. Unsere Entdeckung, dass Dünndarmzellen kleine Mini-Därme generieren können, ${ }^{13}$ gab dann den Anstoß für die Entwicklung der auf adulten Stammzellen basierenden Organoidforschung.

SB: Können Sie sich erinnern, wann Sie das medizinische Potenzial von Organoiden erkannt haben?

HC: Ich glaube, das war direkt, als ich sie das erste Mal gesehen habe. Toshi, Toshiro Sato, der damals Postdoc in meinem Labor war, züchtete sie in Kultur, hatte aber noch niemandem davon erzählt. Ich fragte ihn: „Wie läuft's, züchtest Du schon Darmstammzellen?“ und er antwortete: „Ja, hier sind sie“. Und dann zeigte er sie mir. Im selben Moment, als ich sie sah, wurde mir bewusst: Offensichtlich kann man aus Primärgewebe nicht nur einen Stammzellklumpen züchten - was wir erwartet hatten - sondern tatsächlich ein kleines Organ.

SB: Erwarteten Sie einen Zellklumpen, weil man genau das im iPS-/ES-Zell-Forschungsfeld beobachtet hatte?

12 Eiraku et al., 2008.

13 Sato et al., 2009. 
HC: Ja, das war das, was iPS- und ES-Zell-Forscher damals machten, aus einer Stammzelle eine Million mehr züchten. Einfach nur reine Stammzellen, ohne irgendeine organähnliche Struktur, wie ein Zellhaufen. Unser Ziel war deshalb, dasselbe mit adulten Stammzellen zu machen. Die Zellen machten stattdessen aber etwas anderes, sie organisierten sich zu diesen Mini-Därmen. Wenn man sie wachsen sieht, ist ihre Vitalität auffallend, besonders bei Mauszellen. Das war sehr überraschend. Und als Toshi dann erkannte, dass alle Zelltypen des Darmepithels in diesen Mini-Därmen enthalten waren, wurde uns bewusst, dass man alles, was Forscher damals in Mäusen erforschten, mit menschlichen Zellen machen könnte, sofern es gelingt Organoide auch aus menschlichen Stammzellen zu züchten (was Toshi dann als nächstes tat). Und man kann das sogar für individuelle Patienten machen, auf personalisierte Art. Das war nicht sehr visionär; ich glaube, jeder, der dieses Experiment gesehen hätte, hätte sofort gewusst: regenerative Medizin, Diagnostik, Grundlagenforschung - all dies geht auch mit Organoiden.

SB: Ich erinnere mich an eine Laborbesprechung, als Sie gerade von einer großen Medizinkonferenz zurückgekehrt waren und uns, Ihrer Gruppe, mitteilten, dass ein riesiger Bedarf an besseren Modellen für präklinische Tests bestand.

HC: Zuerst hatten wir die regenerative Medizin im Blick - wir gingen davon aus, dass diese Technologie in erster Linie dazu benutzt würde, Organe nachzubauen und zu reparieren. Doch sehr bald - und zwar ganz plötzlich - entwickelten wir uns von einem regenerativen Labor $\mathrm{zu}$ einem, das an Krankheitsmodellen und prädiktiven Modellen arbeitete. Wir erkannten, dass die regenerative Medizin noch zehn Jahre brauchen würde und dass dies nicht das Gebiet war, auf dem wir als akademisches Labor großartige Beiträge leisten konnten - also stellten wir um auf die Entwicklung von Krankheitsmodellen.

SB: Was außerordentlich produktiv war. Das beste Beispiel ist die Mukoviszidose, bei der Organoide dazu genutzt werden können, vorherzusagen, ob ein Patient auf ein bestimmtes Medikament ansprechen wird oder nicht.

HC: Für Mukoviszidose sind Organoide bereits ein etablierter, integraler Bestandteil des niederländischen Gesundheitssystems. Ich denke, dies ist das erste Mal, dass Organoide offiziell Teil eines Gesundheitssystems geworden sind. Mukoviszidose ist eine Erbkrankheit und in Holland war es vorher so, dass man ein bestimmtes neues Medikament bekam, wenn man die häufigste Mutation hatte.

SB: Warum nur dann, wenn man die häufigste Mutation hatte?

HC: Medikamentenentwicklung und klinische Tests sind sehr teuer, daher hatten die Firmen sich auf die häufigste Mutation spezialisiert. Die seltenen Mutationen wurden nicht in die klinischen Versuche miteinbezogen. 
SB: Es war also unsicher, ob das Medikament bei diesen Patienten anschlagen würde, und es wurde ein Test benötigt, um festzustellen, ob ein bestimmter Patient von der Behandlung profitieren würde?

HC: Und die aus patienteneigenen Zellen entwickelten Organoide ermöglichen genau so einen Test. Heute ist es üblich, dass auch jemand, der eine der vielen seltenen Mutationen hat, sofern der Organoidtest positiv ausfällt, das Medikament erhält und die Kosten dafür von der niederländischen Krankenkasse übernommen werden. Das ging sehr schnell. Die Gründe hierfür waren vielfältig, aber der wichtigste Grund war, dass es kein anderes Medikament für diese Patienten gab. Als wir herausfanden, dass unsere Organoide prognostizieren konnten, ob ein Patient darauf ansprechen würde oder nicht, konnten wir das Medikament tatsächlich den Patienten geben, weil es keine Alternative gab. Es gibt nicht viele andere Erbkrankheiten, bei denen dies der Fall ist. Aber momentan wird viel investiert, um organoidbasierte individuelle Therapien gegen Krebs zu entwickeln. ${ }^{14}$

$\mathrm{SB}$ : Wie könnte das funktionieren?

HC: Ein bisschen wie Antibiotika-Resistenz-Tests bei Bakterien. Wenn man eine bakterielle Infektion hat, werden die Bakterien kultiviert und verschiedenen Antibiotika ausgesetzt. Dann wird das, das am besten wirkt für die Behandlung ausgewählt. Hier würde man nun die Krebszellen als Organoide kultivieren, sie mit verschiedenen Krebsmedikamenten behandeln und dann basierend auf der Reaktion der Organoide das Medikament aussuchen, das für diesen Patienten am besten geeignet ist. Momentan entwickelt sich das sehr schnell. Ich weiß von vielleicht 30 oder 40 laufenden klinischen Beobachtungsstudien.

$\mathrm{SB}$ : Wie laufen diese Studien ab?

HC: Bei Krebs gibt es immer Protokolle und Richtlinien, sodass man nicht sagen kann „Ihr Organoid deutet darauf hin, dass Sie dieses Medikament nicht nehmen sollten, sondern etwas anderes“ - das ist nicht erlaubt. Und es wäre auch nicht klug. Die wissenschaftlichen Belege müssen nach und nach auf eine statistisch aussagekräftige Art und Weise zusammengetragen werden, was gerade parallel an vielen Orten geschieht. In diesen Studien werden Organoide von den Patienten gezüchtet, die Arzneimittelreaktionen an diesen Organoiden getestet und die Reaktion auf die Standardbehandlung bei den jeweiligen Patienten beobachtet und dokumentiert. Dadurch kann man prüfen, ob man diese Reaktion mit den Organoiden hätte vorhersagen können. Die Studie von Giorgios Vlachogiannis in London, die 2018 in der Zeitschrift Science publiziert wurde, zeigte dies sehr gut. 2019 gab es dann einige Artikel zu diesem The-

14 Siehe Kretzschmar, Kap. 3.4. 
ma, auch unter Beteiligung meiner Arbeitsgruppe. ${ }^{15}$ Einer davon, eine Studie unter der Führung von Emile Voest in Amsterdam, ${ }^{16}$ zeigte im Rahmen einer klinischen Studie, dass Organoide sehr gute Vorhersagen ermöglichen. Tatsächlich ist ihre Aussagekraft überraschend hoch, vielleicht weil diese Studien klein sind und die Daten sehr genau angeschaut werden. Das ist sehr vielversprechend.

$\mathrm{SB}$ : Gibt es schon eine Interventionsstudie ${ }^{17}$

HC: Ich weiß, dass man das machen will. Aber dann muss man warten, bis ein Patient aus den Behandlungsrichtlinien herausfällt und solche Patienten sind natürlich nur noch sehr schwer behandelbar. Es bedarf noch sehr viel mehr an Belegen und Evidenz, bevor man die Erlaubnis erhält, einem Patienten nicht die medizinisch bewährte Behandlung zu verabreichen, sondern eine andere, von der man denkt, sie sei besser.

$\mathrm{SB}$ : Was für Belege könnten das sein?

HC: Normalerweise führt man neue Medikamente als Add-on ein, also zusätzlich zur Standardbehandlung. In solchen Studien werden Patienten dem Standard gemäß behandelt, aber dann wird das neue Medikament noch dazu verabreicht. Anschließend wird untersucht, welchen Patienten es besser ergeht. Dadurch wird man sich immer sicherer. Und dann kann man vielleicht etwas von den herkömmlichen Medikamenten reduzieren oder weglassen. Das ist die typische Vorgehensweise. ${ }^{18}$

SB: Bisher ging es um Mukoviszidose und Krebs. Für welche anderen Krankheiten könnten Organoide noch nützlich sein?

HC: Ein Beispiel: Ein Labor in Hong Kong nutzte Atemwegsorganoide, um abzuschätzen, ob neue Grippeerreger gefährlich sind oder nicht. ${ }^{19}$ Neuen Viren, die globale Epidemien auslösen sind häufig Neusortierungen bei denen sich Vogel- oder Schweinegrippeviren mit menschlichen Grippeviren vermischt haben und die häufig aus Südostasien und China stammen. Momentan werden neu auftauchende Virenstämme auf Schnitten menschlichen Lungengewebes getestet, um $\mathrm{zu}$ sehen, ob sie diese infizieren oder nicht. Dabei handelt es sich in der Regel um Gewebeproben die von

15 Etwa Ganesh et al., 2019.

16 Ooft et al., 2019.

17 Eine Interventionsstudie ist eine Studie, bei der prospektiv unter kontrollierten Bedingungen an Patienten die Effektivität einer bestimmten Intervention im Vergleich zu einer Kontrollgruppe untersucht wird. Interventionsstudien folgen während des Entwicklungsprozesses von Medikamenten auf Beobachtungsstudien, die einen explorativen Charakter haben, in der Regel kleiner angelegt sind und Hypothesen generieren.

18 Siehe auch Kretzschmar zu Krebsorganoiden, Kap. 3.4.

19 Zhou et al., 2018. 
Lungenkrebspatienten stammen, und die nicht sehr lange in Kultur gehalten werden können, es ist ein umständliches System. Zwei 2018 publizierte Studien an denen wir beteiligt waren konnten zeigen, dass menschliche Atemwegsorganoide tatsächlich in der Lage sind zwischen Viren, die für Menschen schädlich sind, und solchen, die für Menschen unschädlich sind, zu unterscheiden. Und zwar auf standardisierte Art, weil menschliche Atemwegsorganoide unbegrenzt lange kultiviert werden können, sodass man standardisierte Linien verwenden kann. Ich halte dies für eine sehr wichtige Anwendung. ${ }^{20}$

SB: Das andere große Gebiet, in dem Organoide zur Anwendung kommen, ist die Arzneimitteltoxikologie.

HC: Das Interesse daran ist zurzeit sehr groß. Die Grundidee ist, anstelle von Tieren einfach eine Palette verschiedener Organoide zu benutzen. Etwa Leberorganoide, Nierenorganoide ${ }^{21}$ und Dünndarmorganoide von jeweils zehn Spendern. Derzeit werden neue Medikamente an Tieren getestet, aber Tierversuche erlauben nicht so präzise Vorhersagen wie man vielleicht erwartet. Viele Medikamente bestehen die klinischen Studien in Phase $\mathrm{I}^{22}$ nicht, weil sie für den Menschen toxisch sind, es im Tierversuch aber nicht erkannt wurde.

SB: Das ist ein großes ethisches Problem, aber auch eine finanzielle Frage für die Pharmaindustrie.

HC: Ich bin im engen Gespräch mit dem Leiter eines großen Pharmaunternehmens und er würde Tierversuche am liebsten komplett ersetzen. Er sagt, wir brauchen nur menschliche Modellsysteme. Aber die menschlichen Modelle werden viel mehr von Störfaktoren beeinflusst sein als Tiermodelle. ${ }^{23}$

SB: Wie kommt das?

HC: Selbst wenn man Organoide von nur zehn verschiedenen Probanden gewinnt, erhält man schon sehr unterschiedliche Ergebnisse. Dies liegt natürlich daran, dass diese zehn Individuen einen ganz unterschiedlichen genetischen Hintergrund haben

20 Siehe auch Frum/Spence zu Lungenorganoiden, Kap. 3.1, und Chumduri/Turco zu Infektionsstudien im Urogenitaltrakt, Kap. 3.7.

21 Siehe auch Gupta/Dilmen/Morizane zu Nierenorganoiden, Kap. 3.6.

$22 \mathrm{Zu}$ testende Arzneimittel durchlaufen vor der Zulassung verschiedene Stadien klinischer Tests (Phase 0 bis IV). In Phase-I-Studien wird das Arzneimittel erstmals an einer kleinen Gruppe gesunder Probanden (oder bei bestimmten Indikationen auch Patienten) erprobt, um Informationen über ihre Verträglichkeit und Eigenschaften im Körper zu erhalten.

23 Als Störgeräusche oder Hintergrundrauschen („noise“) bezeichnet man in der Wissenschaft das Phänomen, dass mehr unspezifische Signale erzeugt werden, die herausgefiltert werden müssen, um relevante Signale erkennen zu können. 
und in unterschiedlichen Umgebungen leben. Wenn man hingegen zehn genetisch identische Mäuse nimmt, die unter standardisierten Laborbedingungen leben, erhält man genau dasselbe Ergebnis. Darum lieben Toxikologen die Tiere: die Kurven sehen schön aus. Aber dies spiegelt nicht die Wirklichkeit wider. Daher sind pharmazeutische Firmen sehr daran interessiert, Tiermodelle für Sicherheitstests durch Organoide oder „Organ-on-a-chip“-Modelle zu ersetzen. Bei den Organ-on-a-chip-Modellen werden Zellen oder Organoide in miniaturisierten Zellkulturschalen gehalten und mit winzigen Mengen Nährlösungen versorgt über sogenannte mikrofluidische Systeme. Diese Kombination aus Mikrofluidik und kleinen Zellkultureinheiten wird auch „Lab-on-aChip“ genannt. Ein einzelner Chip ist dabei vielleicht so groß wie der Zeigefinger und Mittelfinger Ihrer Hand zusammen. Viele Ingenieure wollen jetzt diese Chip-Technologie mit Organoiden verbinden und das dann für Medikamententests zur Verfügung stellen. Aber nochmals, neue Tests müssen ihre Überlegenheit erst beweisen.

SB: Wie tragen Sie dazu bei?

HC: Wir forschen daran in der Hubrecht Organoid Technology Foundation, kurz HUB, einer gemeinnützigen Firma, die vom Hubrecht Institut, der Königlich Niederländischen Akademie der Wissenschaften (KNAW) und der Universitätsklinik Utrecht gegründet worden ist. Es gibt eine ganze Reihe von Medikamentenkandidaten pharmazeutischer Firmen, die in Phase I der klinischen Studien gescheitert sind. Das bedeutet, die Medikamente waren bei keinem der Tiere toxisch, bei Menschen jedoch schon. Das HUB hat eine ganze Reihe von Medikamenten erhalten, darunter auch einige dieser Phase-I-Fehlgriffe. Und die Organoide haben diese sofort identifiziert. Das wird nun publiziert. Ich denke, dies ist ein Gebiet, das in den kommenden Jahren sehr viel Aufmerksamkeit erlangen wird.

SB: Das klingt so, als könnten Organoide wirklich Tierversuche für Toxizitätstests komplett ersetzen.

HC: Ja, das Potenzial dazu ist offensichtlich. Das hat verschiedene Gründe. Ein wichtiger Grund ist, dass viele Krankheiten in Mäusen überhaupt nicht gut nachgebildet werden können. Ein weiterer Grund ist ein ethischer: Wenn man Tierversuche vermeiden kann, sollte man es auch tun.

SB: Trotz aller positiven Aspekte der Organoidtechnologie gibt es auch Einschränkungen. Was sind momentan aus Ihrer Sicht die wichtigsten?

HC: Die beiden Technologien, von iPS-/ES-Zellen und von adulten Stammzellen abgeleitete Organoide, sind sehr unterschiedlich. Die Haupteinschränkung unserer Organoidtechnologie, bei der adulte Stammzellen genutzt werden, ist, dass wir ausschließlich Epithelzellen züchten können. Das können wir sehr gut. Wir können dazu Primärgewebe verwenden, man braucht keine reinen Stammzellen, man benötigt nur 
dickes Gewebe, das die Stammzellen enthält. Wir können das für viele Organe machen, aber nicht alle so erzeugten Organoide sind vollständig.

SB: In welcher Hinsicht?

HC: Bei Darm sehen wir zum Beispiel alle Zelltypen, aber beim Magen fehlen uns noch die Parietalzellen. Das muss noch weiterentwickelt werden. Eine weitere Einschränkung unserer Technologie ist, dass wir nur Epithel züchten können, aber nicht Muskeln, Hirn, Knochen oder Fett. Ich bin davon überzeugt, dass diese Gewebe Stammzellen enthalten, die vermutlich anderen Regeln folgen und wahrscheinlich auch andere Kulturbedingungen benötigen, aber es sollte dennoch möglich sein, Knochenorganoide oder Fettorganoide aus adulten Stammzellen zu züchten. Solche Stammzellen, Knochenmarksstammzellen beispielsweise, also die Stammzellen die als erste entdeckt wurden, können nicht in Zellkultur vermehrt werden. Ich bin mir sicher, dass dies ein technisches Problem ist, das gelöst werden kann, aber das ist eine der Einschränkungen: Wir können nur Epithelzellen züchten. Außerdem haben wir keine Mikroben, keine Immunzellen, keine Blutgefäße - die müssen alle von außen hinzugefügt werden.

SB: Und die andere Technologie, die iPS- und ES-Zell-basierten Organoide?

HC: Die können Organoide aus Geweben herstellen, die wir nicht züchten können, insbesondere des Zentralnervensystems ${ }^{24}$ aber auch der Niere. ${ }^{25}$ Die Glomeruli der Niere, die Funktionseinheiten, die die Filterarbeit leisten, haben zum Beispiel keine Regenerationsfähigkeit und können - vermutlich deswegen - nicht als Organoide aus adulten Stammzellen gezüchtet werden. Züchtet man aber Nieren aus iPS-Zellen, können Glomeruli entstehen, weil diese Technologie auf den Mechanismen der Entwicklungsbiologie basiert und nicht auf den Reparaturmechanismen. Wie Jim Wells in Cincinnati zeigen konnte, entwickeln sich nicht alle Zellen exakt entlang der angestrebten Entwicklungslinie, zum Beispiel wenn man versucht, iPS-Zellen in Darmgewebe $\mathrm{zu}$ verwandeln. ${ }^{26}$ Einige bleiben Mesodermzellen, sodass die entstehenden Gewebe vollständiger sind. Dennoch gibt es viele Diskussionen um die Variabilität von iPS- und ES-Zell-basierten Organoiden. Das liegt daran, dass diese Zellen einen langen Weg vor sich haben, bis sie zum Organoid werden. Die Entwicklung kann drei Monate oder länger dauern. Wenn es zu Beginn kleine Unterschiede gibt, werden sie auf diesem langen Weg erheblich amplifiziert. Hat man etwa zehn Mini-Gehirne und untersucht daraus gewonnene Gewebeschnitte, sind sie alle unterschiedlich. Das ist

24 Siehe Tanaka/Park, Kap. 3.5.

25 Siehe Gupta/Dilmen/Morizane, Kap. 3.6.

26 Múnera/Wells, 2017. 
wieder ein rein technisches Problem; das wird vermutlich gelöst werden und Forscher werden besser verstehen, wie man genau die Regionen des Gehirns induziert, die man erzeugen möchte.

$\mathrm{SB}$ : Aber das gilt nur für Organoide, die auf ES-/iPS- Zellen zurückgehen?

HC: Ja. Ich möchte betonen, dass dies nicht für die auf adulten Stammzellen basierenden Organoide gilt. Die sind ziemlich homogen.

SB: Wie nahe kommt denn ein Hirnorganoid an ein Gehirn heran?

HC: Selbst wenn sie ideal sind, sind Organoide immer eine reduktionistische Abstraktion des wirklichen Lebens, eines ganzen Organs oder eines echten Körpers, daher sollte niemand denken, dass ein Hirnorganoid das Gleiche wäre wie ein vollständiges Gehirn. ${ }^{27}$ Keine Sammlung von Organoiden ist mit einer Maus oder einem Menschen äquivalent.

SB: Aber sie können Teil eines Körpers werden, wenn man sie transplantiert. Womit wir bei den ethischen Implikationen der Organoidtechnologie wären. Was halten Sie von Chimären, insbesondere Chimären mit Hirnorganoiden, die erzeugt werden, wenn man menschliche Organoide in Tiere transplantiert?

HC: Es ist äußerst wichtig, sich mit den ethischen Aspekten auseinanderzusetzen. Aber ich glaube nicht, dass Biologen besser in der Lage sind als andere Menschen, sich eine Meinung darüber zu bilden, was wünschenswert ist, was erlaubt werden sollte und was nicht. Ich denke, das was Biologen tun können, ist vor allem die Möglichkeiten aufzuzeigen, die plötzlich entstehen, wenn eine neue Technologie entdeckt wird. Dann müssen gute Ethiker und Rechtsexperten gefunden werden, die in einem geeigneten Verfahren entscheiden, ob wir so etwas tun sollten oder nicht.

$\mathrm{SB}$ : Wie denken Sie persönlich darüber?

HC: Um ehrlich zu sein, Forscher haben menschliche Hirnorganoide in Mausgehirne transplantiert ${ }^{28}$ und da wird mir persönlich etwas mulmig. Ich weiß nicht genau, warum. Das ist nicht unser Forschungsgebiet. Das ist eine Frage, bei der Ethiker mitreden und mithelfen müssen, Regeln des Umgangs zu entwickeln. Ich glaube nicht, dass so ein Ein-Millimeter-Stück menschliches Gehirn ein Bewusstsein entwickeln kann, aber man weiß es auch nicht. ${ }^{29}$

SB: Wie beurteilen Sie Embryoide, also kleine, aus ES- oder iPS-Zellen hergestellte Organoide, die frühe Embryonen nachbilden?

27 Siehe zu Hirnorganoiden auch Tanaka/Park, Kap. 3.5, und Schicktanz, Kap. 6.

28 Mansour et al., 2018.

29 Zu ethischen und kulturellen Aspekten von Mensch-Tier-Chimären siehe Schicktanz, Kap. 6. 
HC: Das ist noch so ein Fall, bei dem mir persönlich ein bisschen unwohl ist. Aber nicht als Wissenschaftler, denn an sich ist das hochspannend. ${ }^{30}$ Aber als Vater vielleicht oder als Bürger wünsche ich mir, dass ausführlich diskutiert wird, ob wir als Gesellschaft das wollen. Weil man dabei tatsächlich Leben erzeugt ohne Spermium und Eizelle. Das ist in dieser Welt so nicht vorgesehen.

SB: Und der weibliche Reproduktionstrakt? ${ }^{31}$ Zum Beispiel die Züchtung eines Plazenta-Organoids, mit dessen Hilfe vielleicht eines Tages ein Embryo heranreifen könnte?

HC: Das finde ich weniger problematisch. In dieser Hinsicht ist die Diskussion um die künstliche Befruchtung (IVF) in den siebziger Jahren sehr interessant. Die Menschen waren damals schockiert von dieser Idee. Inzwischen gibt es Länder, in denen eines von vier Babys durch IVF erzeugt wird und es ist überhaupt kein Thema mehr. Verschiedene Kulturen sehen das auch sehr unterschiedlich. Eine künstliche Gebärmutter ist eher etwas Technisches, das ist nicht annähernd so nah am Kern des Lebens wie ein Embryo, der nicht aus zwei Keimzellen entstanden ist. Oder Mäuse mit einem zum Teil menschlichen Gehirn. Ich denke, das sind ganz unterschiedliche Dinge. Und wir sollten sie diskutieren.

SB: Wenn Sie träumen dürften: Was wird in zwanzig Jahren möglich sein?

HC: In zwanzig Jahren werden Organoide Tierversuche in Toxizitätsstudien ersetzt haben, denke ich. Es wird viele weitere Anwendungsmöglichkeiten von Organoiden geben, um menschliche Reaktionen auf Medikamente oder Infektionen vorherzusagen. Und, wer weiß, in zwanzig Jahren hat man vielleicht auch begonnen, Organoide als Transplantationsmaterial zu nutzen. Wir werden vielleicht dazu in der Lage sein, mithilfe von Organoiden eine Leber oder eine Bauchspeicheldrüse wiederherzustellen. Aber bis dahin müssen wir noch einen weiten Weg zurücklegen.

\subsubsection{Literaturverzeichnis}

Eiraku, M. et al. (2008): Self-organized formation of polarized cortical tissues from ESCs and its active manipulation by extrinsic signals. In: Cell Stem Cell 3(5): 519-32.

Ganesh, K. et al (2019): A rectal cancer organoid platform to study individual responses to chemoradiation. In: Nature Medicine 25: 1607-1614.

Mansour, A. A. et al. (2018): An in vivo model of functional and vascularized human brain organoids. In: Nature Biotechnology 36(5): 432-441.

30 Siehe zu Embryoiden auch Nicolas/Etoc/Brivanlou, Kap. 5.

31 Siehe auch Chumduri/Turco zu Organoiden des weiblichen Reproduktionstraktes (Kap. 3.7). 
Múnera, J. O./Wells, J. M. (2017): Generation of gastrointestinal organoids from human pluripotent stem cells. In: Methods of Molecular Biology 1597: 167-177.

Ooft, S. N. et al. (2019): Patient-derived organoids can predict response to chemotherapy in metastatic colorectal cancer patients. In: Science Translational Medicine 11(513): eaay2574.

Sato, T. et al. (2009): Single Lgr5 stem cells build crypt-villus structures in vitro without a mesenchymal niche. In: Nature 459(7244): 262-265.

Vlachogiannis, G. et al. (2018): Patient-derived organoids model treatment response of metastatic gastrointestinal cancers. In: Science 359(6378): 920-926.

Zhou, J. et al. (2018): Differentiated human airway organoids to assess infectivity of emerging influenza virus. In: Proceedings of the National Academy of Sciences USA 115(26): 6822-6827. 
